\title{
BMJ Open Study protocol: long-term effect of the New Nordic Renal Diet on phosphorus and lipid homeostasis in patients with chronic kidney disease, stages 3 and 4: a randomised controlled trial
}

\author{
Nikita Misella Hansen (D) , ${ }^{1}$ Marianne Rix, ${ }^{1}$ Anne-Lise Kamper, ${ }^{1}$ \\ Bo Feldt-Rasmussen, ${ }^{1}$ Christina Christoffersen, ${ }^{2}$ Arne Astrup, ${ }^{3}$ Louise Salomo ${ }^{1}$
}

To cite: Hansen NM, Rix M, Kamper A-L, et al. Study protocol: long-term effect of the New Nordic Renal Diet on phosphorus and lipid homeostasis in patients with chronic kidney disease, stages 3 and 4 : a randomised controlled trial. BMJ Open 2021;11:e045754. doi:10.1136/ bmjopen-2020-045754

- Prepublication history and additional supplemental material for this paper are available online. To view these files, please visit the journal online (http://dx.doi.org/10.1136/ bmjopen-2020-045754)

Received 12 0ctober 2020 Accepted 17 August 2021

Check for updates

(C) Author(s) (or their employer(s)) 2021. Re-use permitted under CC BY-NC. No commercial re-use. See rights and permissions. Published by BMJ.

${ }^{1}$ Department of Nephrology, Rigshospitalet, Copenhagen, Denmark

${ }^{2}$ Department of Clinical Biochemistry, Rigshospitalet, Copenhagen, Denmark ${ }^{3}$ Department of Nutrition, Exercise and Sports, University of Copenhagen, Copenhagen, Denmark

\section{Correspondence to}

NM Hansen Nikita Misella Hansen;

nikita.misella.hansen@regionh. $\mathrm{dk}$

\section{ABSTRACT}

Introduction Chronic kidney disease (CKD) causes severe disturbances in phosphate metabolism. New Nordic Renal Diet (NNRD) is a new dietary concept designed by the present research group that aims to offer patients with moderate CKD a whole food approach with a markedly reduction in dietary phosphorus intake, corresponding to $850 \mathrm{mg} /$ day. The present protocol describes a randomised controlled trial aiming to test the long-term effects of dietary intervention with NNRD versus a non-restricted habitual diet on important parameters of phosphorus and lipid homeostasis.

Methods and analysis This trial will be executed at the Department of Nephrology, Rigshospitalet, University of Copenhagen, Denmark. Sixty patients aged $\geq 18$ years with CKD stages 3 and 4 (estimated glomerular filtration rate between 15 and $45 \mathrm{~mL} / \mathrm{min}$ ) will be recruited and randomly assigned to the intervention or control group. The other inclusion criterion includes a medically stable condition for at least 2 months prior to the start of the study. Exclusion criteria are treatment with phosphate binders, metabolic disorders that require specific dietary regulation, pregnancy and breast feeding, any types of food allergies or those who are vegans. The observation period is 26 weeks including seven study visits at the outpatient clinic combined with a weekly telephone consultation in both groups. A follow-up visit 3 months after study completion finalises the intervention. The primary outcome is the difference in the change in 24-hour urine phosphorus excretion from baseline to week 26 between the two study groups. Secondary outcomes include changes in phosphate-related and lipid metabolism-related blood and urine biochemistry, blood pressure and body composition. Moreover, we wish to explore adherence to the diet as well as quality of life. Ethics and dissemination The study has been approved by the Scientific Ethical Committee of the Capital Region of Denmark and the Danish Data Protection Agency. The results of the studies will be presented at national and international scientific meetings, and publications will be submitted to peer-reviewed journals.

Trial registration number ClinicalTrials.gov (wwwclinicaltrialsgov) Registry (NCT04579315).

\section{Strengths and limitations of this study}

- This study addresses an important evidence gap, since there is no current dietetic treatment for chronic kidney disease stages 3 and 4 with documented clinical effect.

- The 26-week dietary intervention is a strength regarding the change in lipid metabolism.

- Owing to this type of interventional study, blinding is not possible.

Protocol version The protocol, version 2, has been approved by the Ethical Committee Denmark on 18 September 2020. The protocol has also been approved by Data Protection Regulation and Data Protection Law on 15 September 2020. This study protocol is in accordance with the Standard Protocol Items: Recommendations for International Trials.

\section{INTRODUCTION}

Chronic kidney disease (CKD) is defined by the chronic state of a wide range of kidney diseases. It is associated with a marked risk of cardiovascular disease (CVD) compared with the general population. ${ }^{1}$ The excess cardiovascular morbidity in CKD mainly owes to hyperphosphatemia and changes in mineral metabolism, but also traditional risk factors, such as dyslipidaemia, hypertension and insulin resistance are important parameters. ${ }^{2}$

As CKD progresses, normophosphatemia is maintained by increasing the per nephron urinary phosphorus excretion. ${ }^{3}$ This is stimulated by an increase in fibroblast growth factor 23 (FGF23) and parathyroid hormone (PTH) leading to progressively increasing plasma levels of FGF23 and secondary hyperparathyroidism. ${ }^{4}$ This adaptive compensatory mechanism is obtained until the threshold of toxicity where it becomes maladaptive and 
manifested, hyperphosphatemia develops along with high levels of FGF23 and PTH. ${ }^{5}$ Clinically, hyperphosphatemia is associated with high mortality, vascular calcification, endothelial dysfunction and progression of left ventricular hypertrophy. ${ }^{1}$

The daily dietary intake of phosphorus exceeds the recommended level of $850 \mathrm{mg}$ in many western countries. ${ }^{67}$ The main source of phosphorus is protein-rich food; and in a typical western diet, more than half of the protein load originates from animal protein where the bioavailability is high compared with vegetable sources. Our group has previously developed the New Nordic Renal Diet (NNRD) with a reduced phosphorus content and tested the short-term effect on phosphorus homeostasis in moderate CKD. ${ }^{89}$ NNRD is a modification of the New Nordic Diet (NND), which was developed in 2004 in Copenhagen, Denmark. ${ }^{10}$ NND was designed as a Scandinavian counterpart to the Mediterranean diet and has been investigated in large studies regarding parameters on weight loss among obese individuals, blood pressure, lipid metabolism and blood sugar. The result from the 26-week NND study was a significant greater weight loss in the intervention group $(-4.7 \mathrm{~kg})$ compared with the control group, who followed their habitual diet $(-1.5 \mathrm{~kg})$. Moreover, the intervention group had a significant reduction in systolic and diastolic blood pressure. ${ }^{10}$

NNRD is compared with other well-established international diets as shown in table $1 .{ }^{11}$ The main principles of the NNRD whole food approach are the following:

- Maximum of $850 \mathrm{mg}$ phosphorus/day.

- Protein: $0.8 \mathrm{~g} / \mathrm{kg} /$ day.

- $80 \%$ vegetable products; $20 \%$ animal products.

- Maximum of $5 \mathrm{~g} \mathrm{NaCl} /$ day (table salt).

- Fresh raw products.

- Season oriented.

- Primarily organic food products.

- Fish: at least once a week.

- Vegetarian: at least once a week.

- Wide range of fruits and vegetables.

- Easy to follow in daily practice.

- Rich in flavours.

- Sufficient content of micronutrients and macronutrients.

In a randomised cross-over study of 18 participants with stable CKD stages 3 and 4, 1-week of the NNRD diet was compared with a 1-week control period of habitual diet. NNRD was handed out as packaged meals every third day and it was found that the NNRD reduced the daily phosphate intake with approximately $650 \mathrm{mg} /$ day compared with habitual diet. The 24-hour urine phosphorus excretion was reduced by almost $40 \%$ and a significant $29 \%$ reduction in the 24-hour urinary fractional excretion of phosphorus (FePi). Plasma intact FGF23 was reduced by $18 \%$. Lipids were not measured in the pilot study. ${ }^{9}$

Dyslipidaemia in CKD includes low plasma levels of high-density lipoprotein-cholesterol and low-density lipoprotein (LDL)-cholesterol; whereas levels of triglycerides, apolipoprotein B, lipoprotein(a) $(\mathrm{Lp}(\mathrm{a}))$, intermediate-density and very low-density lipoprotein are often increased. Total cholesterol levels are generally not elevated. ${ }^{2}$ The plasma levels of LDL are well-established risk markers for CVD. However, some investigators challenge this assertion, and have not been able to sustain a link between saturated fatty acids (SFAs) from the diet and CVD risk. ${ }^{12}$ Until recently, SFAs in the diet have been associated with an increased risk of CVD in the general population. However, a recent study suggests that a diet rich in SFA may not in itself increase the risk of CVD, whereas an increase in carbohydrate (primarily from starch and sugar) might be a potential risk factor. ${ }^{13}$ The Mediterranean diet has been shown to reduce the risk of CVD without significantly reducing LDL. ${ }^{14}$ Moreover, a significant reduction in CVD risk by the inhibition of the sodium-glucose cotransporter type 2 has been shown despite a simultaneous increase in LDL levels. ${ }^{15}$ Prospective cohorts have demonstrated that biomarkers of very long chain SFAs were not associated with an overall risk of CVD. It even suggests that high levels of plasma/ serum SFAs might be inversely associated with coronary heart disease. ${ }^{16}$ Therefore, further studies beyond the traditional lipid biomarkers for CVD risk are needed. The NNRD consists of $80 \%$ fruits and vegetables and $20 \%$ animal products, thus having a low content of SFA. Moreover, NNRD has a low content of refined starch and added sugar, which are considered to be risk factors for atherosclerotic events in the general population. It is, therefore, expected that this interventional diet will have a lipid-lowering effect.

The study is developed in collaboration with experts in clinical nutrition and phosphorus and lipid disturbances. The overall objective of this present study is to evaluate the impact of NNRD in patients with moderate CKD over a period of 6 months on the following parameters: (1) phosphorus homeostasis, (2) lipid metabolism and (3) to investigate feasibility, dietary satisfaction and quality of life, measured by the validated questionnaire, EuroQoL 5-Dimension 5-Level (EQ-5D-5L) ${ }^{17} 18$ and in compliance with the diet. To support this, we will also use a validated five-level scale (5-point Likert scale) questionnaire. Participants are able to give five different standardised answers to each question regarding dietary satisfaction and quality of life. If the study is successful, this intervention might reduce the high cardiovascular morbidity in patients with moderate CKD.

\section{METHODS AND ANALYSIS}

\section{Patient and public involvement}

Patients contributed to the design of this study protocol. The project group has conducted interviews with several patients in terms of creating the recipes for the intervention and planning the follow-up visits. The research group designed this study using patients' experiences from the previous mentioned pilot study. ${ }^{9}$ Moreover, the patients will be central during the feasibility state and dissemination upon obtaining results. 
Table 1 Overview of dietary guidance and whole food approach

\begin{tabular}{|c|c|c|c|}
\hline Dietary pattern & Population & Nutrient based & Food based (servings or grams) \\
\hline DASH & $\begin{array}{l}\text { General population, } \\
\text { hypertension }\end{array}$ & $\begin{array}{l}\text { Total fat }(E \%): 27 \\
\text { Saturated fat }(E \%):<6 \\
\text { Protein (E\%): } 18 \\
\text { Potassium (mg): } 4700 \\
\text { Calcium (mg): } 1200 \\
\text { Magnesium (mg): } 450\end{array}$ & $\begin{array}{l}\text { Fish (non-fried): }>2 \text { servings/week } \\
\text { Meat: } \leq 2 \text { servings/day } \\
\text { Nuts/legumes: } 1 / 2 \text { serving/day } \\
\text { Dairy: } 2-3 \text { servings/day } \\
\text { Vegetables: } 4-5 \text { servings/day } \\
\text { Fruits: } 4-5 \text { servings/day } \\
\text { Cereals: ( } \geq 1 / 2 \text { serving whole grain) }\end{array}$ \\
\hline NND & General population & $\begin{array}{l}\text { Total fat }(E \%): 32 \\
\text { Saturated fat }(E \%): 10 \\
\text { Monounsaturated fatty acids (E\%): } 13 \\
\text { Polyunsaturated fatty acids (E\%): } 8 \\
\text { Protein (E\%): } 17 \\
\text { Total carbohydrate }(E \%): 51 \\
\text { Added sugar (E\%): }<10 \\
\text { Fibre }(g): 41 \\
\text { Phosphorus (mg): } 1910 \\
\text { Potassium }(\mathrm{mg}): 5100 \\
\text { Calcium }(\mathrm{mg}): 1400 \\
\text { Magnesium }(\mathrm{mg}): 480 \\
\text { Sodium }(\mathrm{g}): 3\end{array}$ & $\begin{array}{l}\text { Fruits }(g / \text { day }):>300 \\
\text { Vegetables }(g / \text { day }):>400 \\
\text { Nuts }(g / \text { day }):>30 \\
\text { Fish }(g / \text { day) }:>43 \\
\text { Meat }(g / \text { day }):>85-100\end{array}$ \\
\hline
\end{tabular}

DASH, Dietary Approach to Stop Hypertension; NND, New Nordic Diet; NNRD, New Nordic Renal Diet.

\section{Study design and randomisation}

This study is a randomised controlled trial performed at the Department of Nephrology Rigshospitalet, Copenhagen, Denmark. Sixty patients will be randomised to either 26 weeks on the NNRD (intervention) or 26 weeks on their habitual diet (control) (see online supplemental appendix 1). A simple random sample without replacement will be selected by a non-study team member using the lottery method. The study participants can leave the trial at any time during the study period, without any explanation. The investigator can at any time remove a subject from the trial, if there is concern for the patient's safety or if there is a breach in terms of following the protocol. The principal investigator is required to document and report dropouts from the study. Thirty participants will be recruited in each randomisation arm, and a minimum of 21 in each arm must complete the study according to the power calculation.

\section{Study population}

Sixty patients with moderate CKD (stages 3 and 4, estimated glomerular filtration rate (eGFR) $20-45 \mathrm{~mL} /$ 
$\min / 1.73 \mathrm{~m}^{2}$ ) will be recruited from the outpatient clinic at the Department of Nephrology, Copenhagen University, Rigshospitalet. We expect to enrol all 60 participants within 24 months and to complete the study by month 33 .

\section{Inclusion criteria}

- Age $>18$ years.

- eGFR 20-45 mL/min/1.73 $\mathrm{m}^{2}$ as judged by the Chronic Kidney Disease Epidemiology Collaboration (CKD-EPI) equation.

- Medically stable for 2 months prior to start of the study.

- Informed consent form signed after receiving oral and written information.

- Read, speak and understand Danish.

\section{Exclusion criteria}

- Current treatment with phosphate binders.

- Metabolic disorders that require specific dietary regulation.

- Treatment with chemotherapy within the past 6 months.

- Pregnancy and breast feeding.

- Food allergies.

- Vegans.

\section{Intervention}

The project group has entered a cooperation agreement with the Danish chef Claus Meyer and Meyers Madhus. A team of highly talented chefs will create the recipes for the intervention. The recipes will be created in close collaboration with the principal investigator who will use her expertise within clinical nutrition to combine the international nutrients guidelines and culinary experiences. Moreover, the agreement holds that the raw food items and recipes will be delivered in boxes to the participants' homes once a week, throughout the study period. There will be no financial costs for the participants. The intervention group is expected to follow the recipes for 5 consecutive days of the week, the 2 remaining days they must plan their own meals following the guidelines of the NNRD whole food approach.

A 48-hour food record is obtained once a month to control for adherence, and once a week the primary investigator will be in contact by phone providing support and advice on practical questions regarding the diet and checking adherence.

\section{Methods to determine phosphorus intake and homeostasis}

The 24-hour urine collection is the gold standard method for measuring dietary phosphorus. ${ }^{19}$

Measuring urinary excretion of phosphorus, calcium, urea, protein, magnesium, sodium and potassium helps us to asses adherence, and determine progression of CKD and other possible spin-off effects.

The use of FePi in place of normal plasma phosphate can give early information of the kinetics of phosphorus and it is a relatively cheap measurement. FePi expresses the phosphate excreted in percentage of the total filtered phosphate.
In subjects with healthy kidneys, a FePi under $20 \%$ is normal and FePi increases as GFR falls. This is based on the hypothesis that although the diseased kidney contains fewer nephrons, the remaining nephrons are functionally normal. The excretion rate of each functioning nephron increases to maintain phosphorus homeostasis. ${ }^{2021}$

FePi will be calculated according to the following formula:

(urine phosphorus (mmol/L)/plasma phosphorus $(\mathrm{mmol} / \mathrm{L})) \times($ plasma creatinine $(\mathrm{mmol} / \mathrm{L}) /$ urine creatinine $(\mathrm{mmol} / \mathrm{L})) \times 100{ }^{22}$

\section{Trial visits and examinations}

Baseline (day 0)

After oral and written consent has been obtained, randomisation to either the intervention group or control group will be performed by a non-study team member (see online supplemental appendix 2 for an overview of the study timeline). Moreover, the following data will be registered:

- Patient history: gender, age, disease history, current medical record, diagnoses.

- Comorbidities: for example, cardiovascular diseases, chronic obstructive lung disease, diabetes, hyperlipidaemia, hypertension.

- Smoking (years) and alcohol consumption (average per week).

- Level of physical activity (average per week).

- Blood samples: creatinine, eGFR, leucocytes, haemoglobin, lipids, electrolytes, urea, $\mathrm{C}$ reactive protein (CRP), $\mathrm{CO}_{2}, \mathrm{HbA1C}, \mathrm{FGF} 23, \mathrm{PTH}$ and vitamin D.

- 24-hour urinary excretion of phosphorus, calcium, urea, protein, magnesium, sodium, potassium, creatinine clearance.

- DEXA scan.

- ECG.

- Office blood pressure.

- Weight, body mass index (BMI), hip and waist circumference.

- Ankle oedema by subjective clinical assessment.

- Quality of life measured by EQ-5D-5L questionnaire.

- Dietary record: the patients are asked by the study investigator to describe their daily nutritional intake in detail; follow-up questions will be asked in order to get more details regarding the intake.

Day 14

- Blood samples and 24-hour urine collection.

- Blood samples: creatinine, eGFR, leucocytes, haemoglobin, blood lipids, electrolytes, urea, CRP, $\mathrm{CO}_{2}$, FGF23.

- 24-hour urinary excretion of phosphorus, calcium, urea, protein, magnesium, sodium, potassium, creatinine clearance.

- 48-hour dietary record.

Days 30, 60, 90, 120, 150

Every fourth week during the study period of 26 weeks (a total of six times), all participants will be evaluated at the 
Department of Nephrology, University Hospital, Copenhagen. During these first five visits, the following data will be gathered:

- 24-hour urinary excretion of phosphorus, calcium, urea, protein, magnesium, sodium, potassium, creatinine clearance.

- Blood samples: creatinine, eGFR, leucocytes, haemoglobin, blood lipids, electrolytes, urea, $\mathrm{CRP}, \mathrm{CO}_{2}$, FGF23, PTH and vitamin D HbA1C PTH and vitamin D (HbA1C and vitamin D will be measured at day 90).

- Blood pressure.

- Weight, BMI, hip and waist circumference.

- Assessment of ankle oedema by subjective clinical assessment.

- 48-hour dietary record.

\section{Once a week}

Once every week, the principal investigator will offer all the participants a telephone meeting of maximum 20-minute duration evolving on dietary intake and overall conditions. The principal investigator is in close collaboration with the others in the research group who are all medical doctors and will discuss any clinical problem if needed.

\section{Day 180 (study completion)}

During the last day of the study period, the participants will be asked to meet at Rigshospitalet one more time. Data on days 30, 60, 90, 120 and 150 will be gathered, moreover the following information will be obtained:

- 24-hour urinary excretion of phosphorus, calcium, urea, protein, magnesium, sodium, potassium, creatinine clearance.

- Blood samples: creatinine, eGFR, leucocytes, haemoglobin, blood lipids, electrolytes, urea, CRP, $\mathrm{CO}_{2}$, FGF23, HbA1C PTH and vitamin D,

- DEXA scan and ECG.

- Weight, BMI, hip and waist circumference.

- Assessment of ankle oedema by subjective clinical assessment.

- Quality of life measured by EQ-5D-5L questionnaire.

- Dietary satisfaction in the intervention group using a five-level Likert scale.

- Assessment of quality of life in both groups using a five-level Likert scale in combination with the validated EQ-5D-5L questionnaire.

- 48-hour dietary record.

\section{Follow-up visit}

Three months after study completion, there will be an end-of-trial visit with 24-hour urine collection and blood samples (the same markers mentioned at the previous visits). Information about dietary intake will be assessed through 48-hour dietary records. The purpose of this visit is to explore whether the intervention group has been able to follow the guidelines for NNRD after study completion and to examine if there have been any dietary changes in the control group within this period also evaluated by biochemistry analyses of 24-hour urinary collection and blood samples

\section{Research biobank}

For each blood and urine sample, the research group will take out $2 \mathrm{~mL}$ to establish a research biobank. There will be a total of nine blood samples and nine urine samples, which make a total of $18 \mathrm{~mL}$ of blood and $18 \mathrm{~mL}$ of urine for the research biobank (see online supplemental appendix 3). The research biobank will be located at Rigshospitalet. After study completion, the research group wish to explore a series of secondary outcomes, those are described in the Secondary endpoints section.

In case of new research from the blood and urine samples, a new approval from the Scientific Ethical Committee will be needed. Materials in the research biobank will be destroyed after 5 years (approximately 30 July 2025). The materials are only for use within the country and the principal investigator is the main responsible for these.

The trial participants are required to give separate written consent to the research group to store the biological materials in the research biobank (see online supplemental appendix 4).

\section{Outcome measures and analysis methods Primary endpoint}

Difference in change in 24-hour urine phosphorus excretion from baseline to week 26 between the two study groups.

\section{Secondary endpoints}

Difference in changes from baseline to week 26 between study groups in the following:

- 24-hour urinary excretion of phosphorus, calcium, urea, protein, magnesium, sodium, potassium, creatinine clearance.

- Blood samples: blood lipids and electrolytes.

- 24-hour urinary FePi.

- eGFR.

- Weight, hip and waist circumference, and body composition by using DEXA scanning.

- Electrical activity from the heart using ECG.

- Office blood pressure.

- Dietary satisfaction in the intervention group measured by a validated five-level scale (5-point Likert scale) questionnaire.

- Quality of life measured by EQ-5D-5L questionnaire and 5-point Likert scale.

- From the research biobank, the research group wish to explore the following secondary endpoints:

- FGF23.

- Apolipoprotein A1, apolipoprotein B100, apolipoprotein $\mathrm{M}$ and $\mathrm{Lp}(\mathrm{a})$ as part of a substudy on cardiovascular risk.

\section{Sample size calculation and statistical analysis}

The sample size calculation is based on results from previous studies performed by this study group. Within 6 
months of participating in this study, we expect a decrease in eGFR to be about $1 \mathrm{~mL} / \mathrm{min} / 1.73 \mathrm{~m}^{2}$. This will have no influence on the total amount of 24-hour urine phosphorus excretion (primary endpoint), as CKD progresses and normophosphatemia is maintained by increasing the per nephron urinary phosphorus excretion stimulated by an increase in FGF23 and PTH leading to progressively increasing plasma levels of FGF23 and secondary hyperparathyroidism (SHP).

In a randomised controlled study with a type-1 error risk of 0.05 (alpha, two tailed) and a power of $80 \%$ (beta) with an SD of 24-hour urine phosphorus excretion of $200 \mathrm{mg}$ and a minimum clinical relevant difference of $300 \mathrm{mg}$, the study population is estimated at $21 \mathrm{in}$ each group. There will be dropouts so we will include 30 participants in each group. The total number of participants to be included in this study is 60 .

\section{Data analysis}

To compare the biological material (blood and urine) between the two groups over time, we will use a linear mixed model. When comparing the overall results between the intervention and control group, we will use a paired t-test (prerequisite that the data are normally distributed). Non-parametric data will be analysed by the $\mathrm{X}^{2}$ test.

In case that data collection is not complete or in case of insufficient compliance, both intention-to-treat analysis and per-protocol analysis will be performed.

\section{ETHICS AND DISSEMINATION}

\section{Research ethics approval}

In this trial, we expect no serious adverse events or risks. Weight loss is always a concern in nutritional interventions, so the participants will be weighed once a month and DEXA scanning will be performed both at baseline and at the end of the study. The nutritional intervention is not suspected to affect any other medical treatment. All participants participate voluntarily and may at any time withdraw from the trial without it affecting their current or subsequent treatment. The research group can withdraw a patient from the trial at any time if there is a concern for the patient's well-being or if there is a breach in terms of following the protocol. Data on participants who withdraw and protocol breach will be documented. The trial will be performed in accordance with the Helsinki Declaration and national guidelines. The study is approved by the Danish Data Protection Agency and approved by the Ethical Committee of the Capital Region of Denmark. The study is registered on wwwclinicaltrialsgov.

The results of this study will be published in an international journal. The study will have the quality, regardless of a negative or positive result, of being published in a more general high-impact scientific journal. The results will be presented as they are generated at national and international scientific meetings including clinical nutrition and nephrology.
The NNRD study will be presented to the Danish nephrology community and Danish Society for Clinical Nutrition as soon as the protocol is accepted. In close collaboration with the Danish patients' organisation and the Danish Kidney Association, we will develop a strategy for informing their members and patients including meetings for patients and publication in their journal 'Nyrenyt'. The results from the study will also be presented at the Danish Society for Clinical Nutrition and European Society for Clinical Nutrition and Metabolism.

In case of any protocol amendments, the changes will be discussed among the members of the research group and approval will be applied for at the Ethical Committee Denmark.

The participants of the study are covered by University Hospital of Copenhagen health insurance. Moreover, the participants are covered by the law of complaints and compensation within the healthcare system in Denmark. This makes it possible for the participants to fill in complaints to the board of patient complaints and apply for compensation through patient insurance and product liability law.

\section{Data management and data collection plan}

The data capture system Research Electronic Data Capture will be used to create electronic Case Report Forms. All data retrieved during the study will be entered into this system and will be handled according to Good Clinical Practice standards.

The one responsible for the trial will continuously document all data on each patient. All patient information will be processed according to Data Protection Regulation and Data Protection Law. Relevant data can be shared on reasonable request following Data Protection Regulation and Data Protection Law.

\section{Laboratory analyses}

All blood and urine samples will be analysed using standard laboratory methods at the Department of Clinical Biochemistry, Rigshospitalet. Plasma human intact FGF23 will be measured by an FGF23 ELISA kit (Kainos Laboratories, Tokyo, Japan) by batched analyses at the end of the study.

\section{Perspectives}

The perspective of this present study is to investigate the possibility of improving phosphorus and lipid homeostasis in patients with moderate CKD through dietary intervention and to look at feasibility and adherence and to increase patient empowerment. The recipes will be published in a book available for patients with CKD. Furthermore, we aim to make this easily accessible for patients both through patient unions and social media. We anticipate that this study will be of interest to several settings and communities of patients with kidney disease and the professional teams involved in their treatment. This present study may build foundation for a patient 
school involving doctors, nurses and dietitians focusing on dietary prevention in moderate CKD in Denmark.

\section{Uncertainties}

Solid data on long-term treatment effects in CKD stages 3 and 4 regarding the association between dietary intervention and phosphorus homeostasis and lipid metabolism are sparse, therefore the power calculation of the study is associated with uncertainty. The major concern of the present study could be the recruitment of patients and adherence to lifestyle changes. However, according to the study groups' experience from previous clinical trials, patients with CKD are generally highly motivated for participation in studies aimed at improving the knowledge on prevention of progression and treatment of CKD.

Contributors LS, NMH, A-LK and MR designed the study and have taken part in all parts of the protocol. NMH and LS wrote the paper and had primary responsibility for final content. BF-R took part in finalising background and hypothesis, provided support clinically during the study, and critically evaluated the first draft and final version of the manuscripts. He served as the principal supervisor in relation to the Clinical Institute, University of Copenhagen. BF-R, A-LK, MR, AA and CC are all part of the steering committee and contributed to the revision and gave final approval of the paper.

Funding The project has received financial funding from Augustinus Foundation (N/A), Gangsted Foundation (N/A), the Capital Region of Denmark's research foundation (N/A) and the Danish Kidney Association (N/A). The project is fully funded.

Competing interests None declared.

Patient consent for publication Not required.

Provenance and peer review Not commissioned; externally peer reviewed.

Supplemental material This content has been supplied by the author(s). It has not been vetted by BMJ Publishing Group Limited (BMJ) and may not have been peer-reviewed. Any opinions or recommendations discussed are solely those of the author(s) and are not endorsed by BMJ. BMJ disclaims all liability and responsibility arising from any reliance placed on the content. Where the content includes any translated material, BMJ does not warrant the accuracy and reliability of the translations (including but not limited to local regulations, clinical guidelines, terminology, drug names and drug dosages), and is not responsible for any error and/or omissions arising from translation and adaptation or otherwise.

Open access This is an open access article distributed in accordance with the Creative Commons Attribution Non Commercial (CC BY-NC 4.0) license, which permits others to distribute, remix, adapt, build upon this work non-commercially, and license their derivative works on different terms, provided the original work is properly cited, appropriate credit is given, any changes made indicated, and the use is non-commercial. See: http://creativecommons.org/licenses/by-nc/4.0/.

\section{ORCID iD}

Nikita Misella Hansen http://orcid.org/0000-0002-6009-5617
REFERENCES

1 Chen J, Budoff MJ, Reilly MP, et al. Coronary artery calcification and risk of cardiovascular disease and death among patients with chronic kidney disease. JAMA Cardiol 2017;2:635-43.

2 Mikolasevic I, Žutelija M, Mavrinac V, et al. Dyslipidemia in patients with chronic kidney disease: etiology and management. Int J Nephrol Renovasc Dis 2017;10:35-45.

3 Wahl P, Wolf M. Fgf23 in chronic kidney disease. Adv Exp Med Biol 2012;728:107-25.

4 Isakova T, Wahl P, Vargas GS, et al. Fibroblast growth factor 23 is elevated before parathyroid hormone and phosphate in chronic kidney disease. Kidney Int 2011;79:1370-8.

5 Kuro-O M. The FGF23 and klotho system beyond mineral metabolism. Clin Exp Nephrol 2017;21:64-9.

6 Salomo L, Kamper A-L, Poulsen GM, et al. Habitual dietary phosphorus intake and urinary excretion in chronic kidney disease patients: a 3-day observational study. Eur J Clin Nutr $2017 ; 71: 798-800$

7 Institute of Medicine (US), Standing Committee on the Scientific Evaluation of Dietary Reference Intakes. Dietary reference intakes for calcium, phosphorus, magnesium, vitamin D, and fluorideDietary reference intakes for calcium, phosphorus, magnesium, vitamin $D$, and fluoride. Washington, DC: National Academies Press (US), 1997.

8 Salomo L, Poulsen SK, Rix M, et al. The new Nordic diet: phosphorus content and absorption. Eur J Nutr 2016:55:991-6.

9 Salomo L, Rix M, Kamper A-L, et al. Short-Term effect of the new Nordic renal diet on phosphorus homoeostasis in chronic kidney disease stages 3 and 4. Nephrol Dial Transplant 2019;34:1691-9.

10 Poulsen SK, Due A, Jordy AB, et al. Health effect of the new Nordic diet in adults with increased waist circumference: a 6-mo randomized controlled trial. Am J Clin Nutr 2014;99:35-45.

11 Scialla JJ, Lin P-H. Revamping the 'renal' diet: using foods to control phosphorus physiology. Nephrology Dialysis Transplantation 2019;34:1619-22.

12 Mensink RP, Zock PL, Kester ADM, et al. Effects of dietary fatty acids and carbohydrates on the ratio of serum total to HDL cholesterol and on serum lipids and apolipoproteins: a meta-analysis of 60 controlled trials. Am J Clin Nutr 2003;77:1146-55.

13 de Lorgeril M, Renaud S, Mamelle N, et al. Mediterranean alphalinolenic acid-rich diet in secondary prevention of coronary heart disease. Lancet 1994;343:1454-9.

14 Fitó M, Guxens M, Corella D, et al. Effect of a traditional Mediterranean diet on lipoprotein oxidation: a randomized controlled trial. Arch Intern Med 2007;167:1195-203.

15 Scheen AJ. Cardiovascular effects of new oral glucose-lowering agents: DPP-4 and SGLT-2 inhibitors. Circ Res 2018;122:1439-59.

16 Mente A, Dehghan M, Rangarajan S, et al. Association of dietary nutrients with blood lipids and blood pressure in 18 countries: a cross-sectional analysis from the pure study. Lancet Diabetes Endocrinol 2017;5:774-87.

17 Herdman M, Gudex C, Lloyd A, et al. Development and preliminary testing of the new five-level version of EQ-5D (EQ-5D-5L). Qual Life Res 2011;20:1727-36.

18 van Hout B, Janssen MF, Feng Y-S, et al. Interim scoring for the EQ5D-5L: mapping the EQ-5D-5L to EQ-5D-3L value sets. Value Health 2012;15:708-15.

19 Sakuma M, Morimoto Y, Suzuki Y, et al. Availability of 24-h urine collection method on dietary phosphorus intake estimation. J Clin Biochem Nutr 2017;60:125-9.

20 Kaneko I, Tatsumi S, Segawa H, et al. Control of phosphate balance by the kidney and intestine. Clin Exp Nephrol 2017;21:21-6.

21 Slatopolsky E, Robson AM, Elkan I, et al. Control of phosphate excretion in uremic man. J Clin Invest 1968;47:1865-74.

22 Farrow EG, White KE. Recent advances in renal phosphate handling. Nat Rev Nephrol 2010;6:207-17. 